quement indésirable nous conduit inéluctablement à l'idée que ceux qui sont socialement indésirables sont aussi anormaux, et que les causes en sont les mêmes. On dit bien qu'il y a des gènes de l'alcoolisme, de la dépendance envers la drogue, de la pulsion criminelle et de la pauvreté. Mais, si les causes des anomalies individuelles et sociales sont les mêmes, elles relèvent des mêmes traitements. Et si ils ne marchent pas, qu'est-ce qui va marcher ? Le danger de la manie qui consiste à interpréter en termes de biologie moléculaire des maladies individuelles et des problèmes sociaux est que la génétique moléculaire ne peut pas offrir, en tous cas pas beaucoup mieux que la théorie infectieuse des maladies, de possibilité d'éradication de la douleur, de la souffrance et de la mort. Elle peut, tout au mieux, nous permettre d'échanger un type de cancer contre un autre. Le problème n'est pas que des centaines de millions soient dépensés pour le projet "génome humain". Ce n'est pas le projet lui-même qui est en cause, mais la symptomatique d'une explication unique des désordres, des maladies et de la mort. Le vrai problème est que, comme conséquence d'une vaste promesse qui ne peut être tenue, il va en résulter un grand cynisme à l'encontre de la science, et finalement, de la raison. Les gens vont-ils alors en revenir à extirper les yeux?

\section{JEAN TAVLITZKI}

Jean Tavlitzki vient de nous quitter à l'âge de 74 ans.

Il commence, en 1949, sa carrière scientifique de généticien à l'Institut de Biologie Physico-Chimique, dans le domaine de la génétique de la levure, sous la direction du Pr. Boris Ephrussi. Ce travail débouche, en 1958, sur sa thèse de Doctorat ès Sciences intitulée "Recherche génétique et physiologique sur le métabolisme de la Co-carboxylase chez des souches de Saccharomyces cerevisiae exigeant la thiamine ".

Il rejoint ensuite le groupe du Pr. Marianne Manago. En 1960, il quitte son poste de chercheur au CNRS pour occuper les fonctions de prof esseur de génétique à l'Université de Paris ; il y montera un laboratoire de recherche à ce qui deviendra plus tard l'Institut Jacques-Monod. Il étudie alors la différenciation cellulaire du champignon du genre Ustilago cynodontis du chiendent.

C'est à partir de cette époque qu'il se penche sur la didactique de l'enseignement de la biologie et de la génétique dans notre pays. Aussi, à partir de 1982, exerce-t-il une haute responsabilité dans les commissions d'enseignement au Ministère de l'Enseignement Supérieur et occupe-t-il la fonction de conseiller scientifique à la Cité des Sciences et de l'Industrie de la Villette.

L'aboutissement en est : la réforme de l'enseignement de la biologie au lycée, la réalisation d'un merveilleux film didactique "le Code génétique", une bande dessinée "la vie c'est féérique ou La formule magique » éditée en 1984, et de nombreuses cassettes sur douze clefs pour la Biologie en 1985.

Jean Tavlitzki a initié, il y a déjà un quart de siècle, les "Rencontres de Méribel" où de jeunes et de moins jeunes chercheurs et enseignants discutent, d'une manière pluridisciplinaire, des concepts et idées nouvelles dans le domaine de la biologie d'aujourd'hui.

Sa passion pour l'art et la manière d'enseigner la biologie et la génétique, en particulier, a été communiquée à tous ses élèves universitaires qui ont essaimé dans l'enseignement et la recherche. Ainsi, sa mémoire, son message continueront-ils à guider de nombreuses générations d'enseignants et de chercheurs en France.

Animé par sa passion d'enseignant, Jean Tavlitzki était aussi un gentilhomme, amoureux de l'art, de la culture, de la musique pour flûte traversière.

Marc Fellous

\section{R.L. Lewontin}

Professeur à l'Université Harvard, Museum of comparative zoology, Harvard University, 26 Oxford Street, Cambridge, MA 02138, États-Unis. 